\title{
Incorporación del digluconato de clorhexidina como agente inhibidor de las metaloproteinasas en los procesos adhesivos para acrecentar su durabilidad.
}

\section{Incorporation of chlorhexidine digluconate as an inhibiting agent of metaloproteinases in adhesive processes to increase their durability.}

\author{
Patrico Sarmiento-Criollo $^{1 *}$, Stephany Paladines-Calle ${ }^{2}$, Zharina Noblecilla-Alvarado ${ }^{3}$ \\ ${ }^{1}$ Especialista en Odontología Restauradora, \\ 2 Odontóloga de práctica privada, \\ ${ }^{3}$ Egresada de la Carrera de Odontología \\ *drpatsarc@hotmail.com
}

\begin{abstract}
Resumen
El objetivo principal de esta revisión narrativa fue analizar el efecto y estabilidad que ocasiona la incorporación del digluconato de clorhexidina al 0,2 y $2 \%$ en procedimientos restauradores como agente inhibidor de la actividad proteolítica. Para ello se realizó un profundo análisis científico de literatura en el motor de búsqueda pubdmed, para lo cual se utilizó estrategias de búsqueda como el uso de descriptores y operadores booleanos con la finalidad de encontrar información precisa, relevante y actualizada acerca del tema. Evidenciando que la aplicación de digluconato de clorhexidina al $2 \%$ garantiza efectivamente la durabilidad de las restauraciones, preservando la capa hibrida e inhibiendo la actividad de las metaloproteinasas y al mismo tiempo, aumentando considerablemente su estabilidad y longevidad.
\end{abstract}

Palabras clave: Clorhexidina, dentina, durabilidad, sistemas adhesivos..

\begin{abstract}
The main objective of this narrative review was to analyze the effect and stability caused by the incorporation of 0.2 and $2 \%$ chlorhexidine digluconate in restorative procedures as an inhibitor of proteolytic activity. For this, a thorough scientific analysis of the literature was carried out in the pubdmed search engine, for which search strategies such as the use of descriptors and Boolean operators were used in order to find accurate, relevant and updated information on the subject. Evidence that the application of $2 \%$ chlorhexidine digluconate effectively guarantees the durability of the restorations, preserving the hybrid layer and inhibiting the activity of metalloproteinases and at the same time, considerably increasing their stability and longevity.
\end{abstract}

Key words: Chlorhexidine, dentine, durability, adhesive systems..

\section{INTRODUCCIÓN}

En los últimos años ha aumentado el interés clínico sobre la conservación de la estructura dentaria en los tratamientos restauradores. Se han innovado varias técnicas y materiales con la finalidad de lograr biocompatibilidad entre el material y el remanente dentario y conseguir una máxima conservación de los tejidos dentarios. Al hablar de los sistemas adhesivos convencionales, se ha evidenciado nuevas estrategias clínicas que permiten alcanzar una excelente unión del material restaurador con la estructura dentinaria. Estas estrategias consisten en la desmineralización del tejido dentinario por medio de la aplicación de un ácido con la finalidad de exponer las fibras de colágeno, las cuales serán infiltradas por monómeros resinosos que posteriormente se polimerizaran y se dará lugar a la formación de la capa híbrida. ${ }^{1,2}$

Desafortunadamente en varias ocasiones no se logra infiltrar completamente las fibras de colágeno por los monómeros resinosos quedando zonas de dentina desmineralizada por debajo de la restauración, lo cual genera con el paso del tiempo una degradación de las uniones resina-dentina. Las fibras de colágeno que quedaron por debajo de la restauración pueden ser degradas por la acción de las metaloproteinasas (MMPs) que son enzimas 
proteolíticas endógenas pertenecientes al tejido dentinario. ${ }^{3-5}$

Se ha evidenciado que la función que ejercen estas enzimas proteolíticas es la degradación de la matriz extracelular tanto en procesos fisiológicos como en los procesos patológicos. Dentro del tejido dentinario se ha identificado cuatro tipos de metaloproteinasas (MMP-2 y MMP-9 (gelatinasas), MMP-8 (colagenasa), y MMP-20 (enamelisina)). ${ }^{6}$ Las Metaloproteinasas de matriz dentinaria (MMPs), constituyen un factor asociado a la disminución de la longevidad de las restauraciones, ya que son responsables de la degradación del colágeno. ${ }^{7}$

Actualmente se han introducido a los protocolos de adhesión de grabado total, la aplicación y frotación de clorhexidina embebida por quince o treinta segundos en el fondo de la cavidad previa a su restauración, este efecto desinfectante elimina potencialmente las bacterias que podrían irritar el tejido pulpar provocando sensibilidad postoperatoria y caries secundaria, además disminuye la degradación de la capa híbrida, inhibiendo la actividad proteolítica de las metaloproteínasas garantizando de manera directa la longevidad de las restauraciones. ${ }^{8-10}$

\section{ESTADO DEL ARTE}

En el año 1940 en Inglaterra fue desarrollada la formulación de la clorhexidina y para el año 1954 esta fue introducida en el mercado como antiséptico para las heridas de la piel. Siendo altamente empleada en el campo de la medicina durante las intervenciones quirúrgicas utilizándola en la preparación prequirúrgica de la piel tanto del paciente como del profesional. ${ }^{11}$

En el campo de la odontología durante las primeras décadas la clorhexidina fue utilizada únicamente para desinfectar la cavidad bucal. Gracias a estudios desarrollados por Loe y Schiott se comenzó a emplear la clorhexidina como colutorio dental, estos estudios demostraron que utilizando esta formulación durante 60 segundos dos veces al día, en ausencia de una rutina de higiene normal se logró inhibir la formación de la biofilm dental. ${ }^{12,13}$ Baker y colaboradores en el año 1975 consideraron viable el uso de la clorhexidina como irrigante en procedimiento endodónticos. A su vez Delany y colaboradores en el año 1982 comprobaron lo descrito por Baker describiendo que la clorhexidina es un efectivo agente antibacteriano durante la terapia endodóntica. ${ }^{11}$

La principal característica de la clorhexidina es su propiedad de sustantividad, logrando la retención de aproximadamente el $80 \%$ de solución, lo cual indica el tiempo y la calidad de contacto entre el antiséptico y el tejido dentinario. ${ }^{14}$ Gracias a las propiedades catiónicas que presenta la clorhexidina, esta formulación logra unirse a la placa dental a través de la hidroxiapatita del esmalte. La clorhexidina absorbida se libera durante más de 24 horas, lo cual reduce la colonización bacteriana en las superficies dentales. ${ }^{15}$ Webster y colaboradores realizaron un estudio in vitro en el cual demostraron que la clorhexidina posee un amplio espectro antibacteriano que se extiende hasta por 168 horas posteriores a su aplicación. ${ }^{16}$ Esta solución antibacteriana es relativamente no tóxica, no afecta ni a corto ni a largo plazo a la integridad de los cementos selladores. Posee un amplio espectro de microorganismos, incluyendo el E. faecalis y C. albicans; sin embargo, para que ejerza su acción sobre estos microorganismos la clorhexidina debe ser aplicada al menos al $1 \%$ pero preferentemente debe ser aplicada al $2 \% .^{17,18}$

En el año 1999, Gendron y su equipo de contribuyentes investigativos revelaron que la clorhexidina utilizada a muy bajas concentraciones lograba inhibir parcialmente la actividad proteolítica de la MMP-2, MMP-8 y MMP-9. En el caso de la MMP-2 y MMP-9, el efecto inhibitorio se genera por la producción de mecanismos de quelación y atrapamiento de ciertos iones metálicos, especialmente iones cálcicos y iones zinc, lo que provoca la interrupción e impedimento de la activación y reacción catalítica. A su vez el gluconato de clorhexidina interacciona o ataca los grupos sulfhídrilos de las proteínas e induce reacciones de cambio de cisteína involucradas tanto en la activación como en su inhibición MMP-8. ${ }^{19}$

\section{- Uso de la clorhexidina en el protocolo de sistemas adhesivos convencionales:}

En el año 2004 Pashley y colaboradores realizaron un estudio in vitro utilizando matrices de dentina desmineralizada, las cuales fueron almacenas en un medio salival artificial durante 250 días. Llegando a la conclusión que el tejido dentinario sano posee la capacidad de degradar las fibras de colágeno expuestas por el grabado ácido en ausencia de microrganismos bacterianos. Esta acción se genera debido a las metaloproteinasas liberadas a lo largo del tiempo en el huésped, además indicaron que debido al uso de clorhexidina a $0,2 \%$ por 60 segundos retardó el proceso de degradación de estas fibras de colágeno expuestas. Recomendó que se debe utilizar este agente antibacteriano sobre la dentina grabada previo a la aplicación de adhesivos de grabado y lavado. ${ }^{20}$

En el año 2006 se introdujo un protocolo específico para evitar la degradación de la capa híbrida. Heblingy y colaboradores incorporaron al gluconato de clorhexidina al protocolo de aplicación de un adhesivo convencional durante un procedimiento restaurador. ${ }^{21}$ Utilizaron cierta muestra de diente deciduos que presentaban lesiones cariosas contralaterales, luego de la remoción del tejido cariado, 
fue aplicado el adhesivo en un lado de la lesión siguiendo las instrucciones del fabricante y en el otro lado aplicaron clorhexidina al $2 \%$ después de la incorporación del grabado ácido y anterior a la aplicación de el adhesivo convencional. Luego de seis meses fueron analizados mediante microscopia electrónica de transmisión demostrándose que las piezas dentales en las que se aplicó el agente antibacteriano mostraron una región adhesiva en óptimas condiciones, mientras que en las piezas dentales que no se aplicó clorhexidina al $2 \%$ se detectó desintegración progresiva de la red de fibras de colágeno. ${ }^{21}$

En una investigación realizada por Brackett y colaboradores tomaron como muestra premolares permanentes libres de caries, clasificándolos en dos grupos, un grupo control (sin clorhexidina) y un grupo experimental (con clorhexidina). En el grupo experimental se aplicó glucanato de clorhexidina al $2 \%$ posterior a la aplicación del grabado ácido y anterior a la aplicación del adhesivo. Para el análisis de la evolución de estas piezas dentales se utilizó microscopia electrónica de transmisión observándose degradación de la capa híbrida en las piezas del grupo control y por el contrario en el grupo experimental no se presentó degradación. ${ }^{22,23}$

Brackett $\mathrm{M}$ y colaboradores realizaron un estudio similar a los anteriores con la diferencia que utilizaron un adhesivo a base de acetona, al contrario de los estudios pioneros donde el adhesivo empleado fue a base de etanol. Las piezas dentales utilizadas en este estudio fueron premolares sanos. Mediante microcopia electrónica de transmisión se llegó a la conclusión que en el grupo control se experimentó una degradación extensa mientras que el grupo experimental no se observó degradación. ${ }^{24,25}$

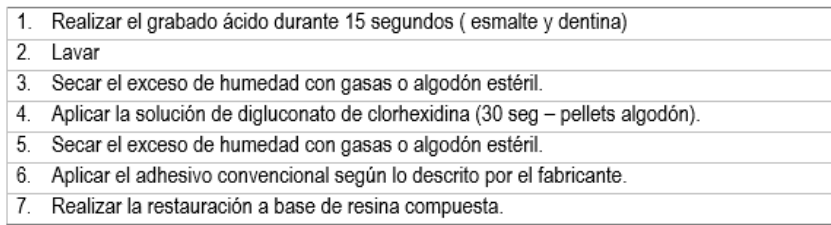

Tabla 1. Protocolo de aplicación de adhesivos convencionales usando clorhexidina.

\section{- Mecanismo de acción de la clorhexidina en preserva- ción de la capa hibrida:}

En un estudio realizado por Pashley, se demostró que la acción de las MMPs fue inhibida por el uso de clorhexidina, ayudando de esta manera a preservar la integridad estructural de las fibrillas de colágeno, lo cual minimizaría la degradación de la capa híbrida. ${ }^{26}$

Turner y colaboradores revelaron que las actividades de degradación del colágeno dentinario pueden reducirse mediante el uso de digluconato de clorhexidina sobre la superficie de la dentina después de la aplicación de ácido fosfórico y antes del adhesivo. ${ }^{27}$ Además de ello se ha constatado que la solución de digluconato de clorhexidina es capaz de inhibir completamente la actividad de MMP-2 y MMP-9, incluso en concentraciones tan pequeñas como al $0,03 \% .^{28}$

La clorhexidina posee un amplio espectro de acción ante microorganismos Gram negativos y Gram positivos, siendo elemental la aplicación y su uso en el campo odontológico por la capacidad de contrarrestar y minimizar la formación de la placa dental subsecuente. ${ }^{15}$

La propiedad de sustantividad que posee la clorhexidina ha evidenciado que es altamente efectiva en ciertos tratamientos periodontales como agente antibacteriano inhibiendo la formación de la placa subgingival. ${ }^{29}$ Hammed señala la importancia de la aplicación de clorhexidina al $2 \%$ durante la preparación cavitaria previa a su adhesión con sustratos resinosos inhibiendo e impidiendo la estampa de $S$. mutans que son evidentes en fisuras oclusales y en superficies radiculares expuestas, además la aplicación de esta solución se logra prevenir la caries residual y la sensibilidad postoperatoria. ${ }^{30,31}$

\section{DISCUSIÓN}

Es clara la actividad proteolítica que ejerce el digluconato de clorhexidina durante los procesos de adhesión de grabado total, la aplicación de este agente estabiliza y brinda longevidad a las restauraciones. Singh et al; señalaron que la conservación y preservación de las restauraciones es mucho mayor utilizando la clorhexidina al $2 \%$ en relación con su aplicación al $0,2 \%{ }^{32}$

Por otra parte, Breschi et al; indicaron que la aplicación de clorhexidina al $2 \%$ disuade la incorporación de las metaloproteinasas durante la adhesión al tejido dentinario tanto en las piezas dentales temporales como en permanentes. Además, lograron evidenciar que el digluconato de clorhexidina al $0,2 \%$ ejerce un rol protector demostrando que la eficacia de la clorhexidina no varía según la concentración en la que se la utilice. ${ }^{15}$

Bolhari et al; en su estudio acerca de la efectividad de la aplicación de la clorhexidina al protocolo restaurador de grabado total, evidenciaron los efectos que brinda la clorhexidina al $2 \%$, sobretodo evitando la degradación de las fibrillas del colágeno cuando es aplicado previo al acondicionamiento ácido. ${ }^{2}$

Asimismo, Abu et al; concluyen que la aplicación de clorhexidina al $0,2 \%$ conserva las propiedades de 
resistencia de los tejidos durante la adhesión. ${ }^{31}$ Carrilho y cols; observaron que cuando se aplica la clorhexidina al $2 \%$ luego del grabado ácido, preservara la estabilidad de la capa híbrida y la resistencia de la restauración. ${ }^{33}$

De acuerdo al análisis bibliográfico realizado se logra establecer que la aplicación de la clorhexidina a diversas concentraciones, proporciona iguales resultados, ya que ambas concentraciones logran inhibir la actividad proteolítica de las MMP-2, -8 y -9; logrando conservar la resistencia de la restauración. ${ }^{34}$

Con respecto al momento de aplicación, el digluconato de clorhexidina puede ser aplicado en varios tiempos, se puede aplicar antes de realizar el grabado ácido total, durante la aplicación del ácido fosfórico y después del grabado ácido. Estudios demuestran que la resistencia de unión resina-dentina no se ve afectada por el momento en el cual es aplicada la clorhexidina dentro del protocolo de adhesión. ${ }^{35}$

\section{CONCLUSIÓN}

Luego de realizar un amplio análisis bibliográfico, se puede concluir que la incorporación del digluconato de clorhexidina al protocolo restaurador de grabado total, proporciona excelentes resultados conservadores tanto en los tejidos dentarios como en las uniones resina-dentina o interfaz.

Se evidencio que existe un gran número de estudios que constatan y evidencian estadísticamente la efectividad de la clorhexidina al $2 \%$ y un escaso número de revisiones científicas sobre los efectos que proporciona la clorhexidina al $0,2 \%$. Sin embargo, algunos autores manifiestan que la efectividad de la acción del digluconato de clorhexidina no varía en relación a la concentración en la que se la utilice.

Estableciendo que la incorporación de clorhexidina brinda una elevada resistencia en los procesos adhesivos con técnica de grabado total; así mismo, estudios paralelos argumentan que la aplicación de esta sustancia evita y disminuyen la resistencia de las metaloproteínasas. Por lo que, la incorporación del digluconato de clorhexidina, ha evidenciado aumentar la longevidad de las restauraciones, produciendo una mejor fijación sobre la dentina, además de prolongar la degradación de fibras colágenas de la capa híbrida expuesta; lo que genera una región adhesiva con total integridad en la estructura normal de la red colágena, conllevando a su vez al aumento en su estabilidad y longevidad.

\section{Conflicto de Intereses}

Los autores declaran no tener conflicto de intereses.

\section{Referencias Bibliográficas}

1 Mobarak E, Shabayek M, El-Deeb H, Mulder J, Hassan F, Van der Sanden W, et al. Survival of occlusal ART restorations using high-viscosity glass-ionomer with and without chlorhexidine: A 2-year split-mouth quadruple-blind randomized controlled clinical trial. Journal of Advanced Research. 2019; 17:117-123.

2 Pashley D, Tay F, Yiu C, Hashimoto M, Breschi L, Carvalho $\mathrm{R}$, Ito $\mathrm{S}$ Collagen degradation by host-derived enzymes during aging. J Dent Res. 2004; 83(3):216-221.

3 Vallabhdas A, Kumar V, Kabbinale P, Nayak R, Rajakumari M, Shilpa T. Evaluation of Hybrid Layer and Bonding Interface after Water Storage with and without the Usage of $2 \%$ Chlorhexidine: A Scanning Electron Microscope Study. J Contemp Dent Pract. 2018; 19(1):52-59.

4 Yiu C, Hiraishi N, Tay F, King N. Effect of chlorhexidine incorporation into dental adhesive resin on durability of resin-dentin bond. J Adhes Dent. 2012 Aug; 14(4):355-362.

5 Osario R, Yamauti M, Ruiz M, Toledano M. MMPs activity and bond strength in deciduous dentine-resin bonded interfaces. J Dent. 2013; 41(6):549-555.

6 Hamdan-Nassar T, Bellot-Arcís C, Paredes-Gallardo V, García-Sanz V, Pascual-Moscardó A, Almerich-Silla J, Montiel-Company J. Effect of $2 \%$ Chlorhexidine Following Acid Etching on Microtensile Bond Strength of Resin Restorations: A Meta-Analysis. Medicina. 2019; 55(12):769.

7 Klont B, ten Cate J. Susceptibility of the collagenous matrix from bovine incisor roots to proteolysis after in vitro lesion formation. Caries Res. 1991; 25(1):46-50.

8 Pavlík V, Sojka M, Mazúrová M, Velebný V. Dual role of iodine, silver, chlorhexidine and octenidine as antimicrobial and antiprotease agents. PLOS ONE. 2019; 14(1):e0211055.

9 Collins J, Olsen J, Cuesta A, Silva-Vetri M, Hernández M. In vitro microbiological analysis on antibacterial, anti-inflammatory, and inhibitory action on matrix metalloproteinases- 8 of commercially available chlorhexidine digluconate mouth rinses. Indian J Dent Res. 2018; 29(6):799-807.

10 Kawasaki F, Featherstone J. Effects of collagenase on root demineralization. J Dent Res. 1997; 76(1):588-595.

11 Dumas J, Hurion N, Weil R, Keil B. Collagenase in mineralized tissues of human teeth. FEBS Lett. 1985; 187(1):5155.

12 Varma R, Sajil J, Deepika V, Dwijendra K, Ram B, Chincholi S. Eficacia antiplaca del enjuague bucal a base de hierbas y gluconato de clorhexidina al $0,2 \%$ : un estudio comparativo. J Int Salud Bucal. 2015; 7(8):98-102.

13 Fordal O, Turnbull R. A review of the literature on use of chlorhexidine in dentistry. J Am Dent Assoc. 1986; 112(6):863-869.

14 Macías J, Arreguin V, Muñoz J, Álvarez J, Mosqueda J, Macías A. La clorhexidina es un mejor antiséptico que la 
povidona yodada y el hipoclorito de sodio debido a su efecto sustancial. Soy J Infect Control. 2013; 41(7):634-637.

15 Weber C, McClanahan S, Miller G. Diener M, Johnson J. 27 The effect of passive ultrasonic activation of $2 \%$ chlorhexidine or $5.25 \%$ sodium hypochlorite irrigant on residual antimicrobial activity in root canals. J Endod. 2003; 29(9):562564.

16 Strand S, Vollmer P. van den Abeelen L, Gottfried D, 28 Alla V, Heid $\mathrm{H}$, et al. Cleavage of CD95 by matrix metalloproteinase-7 induces apoptosis resistance in tumour cells. Oncogene. 2004; 23(20):3732-3736.

17 Overall C, Lopez C. Strategies for MMP inhibition in can- 29 cer: innovations for the post-trial era. Nat Rev Cancer. 2002; 2(9):657-672.

18 Ferguson J, Hatton J, Gillespic M. Effectiveness of intracanal irrigants and medications against the yeast Candida albicans. J Endod. 2002; 28(2):68-71.

19 Chaussain C, Fioretti F, Goldberg M, Menashi S. The role of 31 matrix metalloproteinases (MMPs) in human caries. J Dent Res. 2006; 85(1):22-32.

20 Tjäderhane L, Salo T, Larjava H, Larmas M, Overall C. A 32 novel organ culture method to study the function of human odontoblasts in vitro: gelatinase expression by odontoblasts is differentially regulated by TGF-beta1. J Dent Res. 1998; 77(7):1486-1496.

21 Nagase H, Woessner J, Matrix metalloproteinases. J Biol Chem. 1999; 274(31):21491-21494.

22 Delany G, Patterson S, Miller C, Newton C. The effect of 34 chlorhexidine gluconate irrigation on the root canal flora of freshly extracted necrotic teeth. Oral Surg Oral Med Oral Pathol. 1982; 53(5):518-523.

23 Capelli J, Kantarci A, Haffajee A, Teles R, Fidel R, Figuere- 35 do C. Matrix metalloproteinases and chemokines in the gingival crevicular fluid during orthodontic tooth movement. Eur J Orthod. 2011; 33(6):705-711.

24 Brew K, Nagase H. The tissue inhibitors of metalloproteinases (TIMPs): an ancient family with structural and functional diversity. Biochim Biophys Acta. 2010; 1803(1):55-71.

25 Al-Azri A, Gibson R, Keefe D, Logan R. Matrix metalloproteinases: do they play a role in mucosal pathology of the oral cavity?. Oral Dis. 2013; 19(4):347-359.

26 Cheng Xx, Fang H, Xu W. Advances in assays of matrix metalloproteinases (MMPs) and their inhibitors. J Enzyme Inhib Med Chem. 2008; 23(2):154-167.

7 Turner D, Marfurt C, Sattclbcrg C. Demonstration of physiological barrier between pulpal odontoblasts and its perturbation following routine restorative procedures: a horseradish peroxidase tracing study in the rat. J Dent Res. 1989; 68(8):1262-1268.

Sassone L, Fidel R, Fidel S, Vieira M, Hirata R. The influence of organic load on the antimicrobial activity of different concentrations of $\mathrm{NaOCl}$ and chlorhexidine in vitro. Int Endod J. 2003; 36(12):848-852.

9 Trojanek J. Matrix metalloproteinases and their tissue inhibitors. Postepy Biochem. 2012; 58(3):353-362.

30 Loguercio A, Stanislawczuk R, Polli L, Costa J, Michel $\mathrm{M}$, Reis A. Influence of chlorhexidine digluconate concentration and application time on resin-dentin bond strength durability. Eur J Oral Sci. 2009; 117(5):587-596.

Birkedal H, Moore W, Bodden M, Windsor L, Birkedal B, DeCarlo A, et al. Matrix metalloproteinases: a review. Crit Rev Oral Biol Med. 1993; 4(2):197-250.

Gendron R, Grenier D, Sorsa T, Mayrand D. Inhibition of the activities of matrix metalloproteinases 2,8 , and 9 by chlorhexidine. Clin Diagn Lab Immunol. 1999; 6(3):437439.

33 Balandrano R. Soluciones para irrigación en endodoncia: hipoclorito de sodio y gluconato de clorhexidina. Revista Científica Odontológica. 2007; 3(1):11-14.

4 Lambrechts P, Van Meerbeek B, Perdigao J, Vanherle G. Adhesives: Dos and Don'ts In: Roulet JF. Degrange M. Adhesion: The silent revolution in dentistry. Quintessence. 2000; 4:45-60.

5 Brackett F, Tay M, Brackett A, Dib R, Pashley D. The Effect of Chlorhexidine on Dentin Hybrid Layers In Vivo. Operative Dentistry. 2007; 32(2):107-111.

Recibido: 20 de Febrero del 2020

Aceptado: 19 de Agosto del 2020 
